\title{
Glucose transporter-1 in pulmonary neuroendocrine carcinomas: expression and survival analysis
}

\author{
Irem H Ozbudak ${ }^{1}$, Konstantin Shilo², Fabio Tavora ${ }^{2}$, Negar Rassaei ${ }^{2}$, Wei-Sing Chu $^{3}$, \\ Junya Fukuoka ${ }^{4}$, Jin Jen ${ }^{5}$, William D Travis ${ }^{6}$ and Teri J Franks ${ }^{2}$ \\ ${ }^{1}$ Department of Pathology, University of Akdeniz School of Medicine, Antalya, Turkey; ${ }^{2}$ Department of \\ Pulmonary and Mediastinal Pathology, Armed Forces Institute of Pathology, Washington, DC, USA; \\ ${ }^{3}$ Department of Scientific Laboratories, Armed Forces Institute of Pathology, Washington, DC, USA; \\ ${ }^{4}$ Laboratory of Pathology, Toyama University Hospital, Toyama, Japan; ${ }^{5}$ Laboratory of Human \\ Carcinogenesis, Center for Cancer Research, National Cancer Institute, Bethesda, MD, USA and \\ ${ }^{6}$ Department of Pathology, Memorial Sloan Kettering Cancer Center, New York, NY, USA
}

\begin{abstract}
Glucose transporter-1 (GLUT-1) mediates the transport of glucose across the cellular membrane. Its elevated levels and/or activation have been shown to be associated with malignancy. The aim of this study was to investigate GLUT-1 expression in pulmonary neuroendocrine carcinomas. Tissue microarray-based samples of 178 neuroendocrine carcinomas, including 48 typical carcinoids, 31 atypical carcinoids, 27 large cell neuroendocrine carcinomas and 72 small cell carcinomas from different patients, were studied immunohistochemically for GLUT-1 expression. Forty-seven percent (75/161) of pulmonary neuroendocrine carcinomas were immunoreactive with GLUT-1. GLUT-1 was observed in 7\% (3/46) of typical carcinoid, 21\% (6/29) of atypical carcinoid, $74 \%(17 / 23)$ of large cell neuroendocrine carcinoma and $78 \%(49 / 63)$ of small cell carcinoma. GLUT-1 expression correlated with increasing patient age $(P=0.01)$ and with neuroendocrine differentiation/tumor type $(P<0.001)$, but not with gender, tumor size or stage. GLUT-1 expression was seen in a characteristic membranous pattern of staining along the luminal borders or adjacent to necrotic areas. GLUT-1 expression was associated with an increased risk of death for neuroendocrine carcinomas as a group (risk ratio = 2.519; 95\% confidence interval $=1.519-4.178 ; P<0.001$ ) and carcinoids (risk ratio $=4.262 ; 95 \%$ confidence interval $=1.472-12.343 ; P=0.01$ ). In conclusion, GLUT-1 is expressed in approximately half of the pulmonary neuroendocrine carcinomas and shows a strong correlation with neuroendocrine differentiation/grade, but not with other clinicopathologic variables. Further studies appear plausible to elucidate the prognostic significance of GLUT-1 expression in pulmonary carcinoids.
\end{abstract}

Modern Pathology (2009) 22, 633-638; doi:10.1038/modpathol.2009.6; published online 20 February 2009

Keywords: GLUT-1; neuroendocrine carcinoma; carcinoid; survival; lung

Neuroendocrine carcinomas of the lung are a distinct subset of pulmonary neoplasms that share common neuroendocrine morphology and immunohistochemical characteristics. They consist of typical carcinoids, atypical carcinoids, large cell

Correspondence: Dr K Shilo, Department of Pulmonary and Mediastinal Pathology, Armed Forces Institute of Pathology, 6825 16th Street NW, Washington, DC 20306, USA.

E-mail: shilok@afip.osd.mil

The opinions and assertions contained herein are the private views of the authors and are not to be construed as official or reflecting the views of the Departments of the Army or Defense. This is a US Government work and, as such, is in the public domain in the United States of America.

Received 14 November 2008; revised 16 January 2009; accepted 20 January 2009; published online 20 February 2009 neuroendocrine carcinomas and small cell carcinomas. Neuroendocrine carcinomas are categorized based on the mitotic rate, presence or absence of necrosis, cell size and nuclear features. ${ }^{1}$ They vary significantly in outcome, as small cell carcinoma has a median survival time of 2-4 months without treatment, whereas typical carcinoid rarely metastasizes and has an excellent prognosis., ${ }^{1,2}$

It is well recognized that malignant cells have accelerated metabolism, high glucose requirement and increased glucose uptake compared with healthy cells. Transmembrane passage of glucose mediated by specific transporters is considered to be one of the most important mechanisms for enhancing glucose influx into cells. Facilitative glucose transporters (GLUTs) allow the energy-independent 
transport of glucose across the hydrophobic cell membrane, down its concentration gradient. ${ }^{3}$ They are known as $\mathrm{Na}^{+}$-independent glucose transporters and they are activated by hypoxia, growth factors, calcium, thyroid hormone, oncogenic transformation and inhibition of oxidative phosphorylation. ${ }^{4}$ Fourteen members of the mammalian facilitative glucose transporter family have been identified: GLUT-1-12, -14 and the H+/myo-inositol transporter. GLUT-1 can be identified only in a limited number of normal tissues, including erythrocytes, germinal cells of the testis, renal tubules, perineurium of peripheral nerves, endothelial cells in bloodbrain barrier vessels and placenta (trophoblasts and capillaries).,5,6 In contrast, elevated levels of GLUT-1 expression and/or activation have been shown to be associated with a variety of carcinomas such as those of the breast, head and neck, bladder, colorectal and lung. ${ }^{3,7-13}$ Overexpression of GLUT is commonly observed in cancer, which is associated with high metabolism and rapid cell growth in often hypoxic tumor regions. ${ }^{14}$ Although most of the GLUT subtypes have been detected in different human cancers, GLUT-1 is the only subtype that has been shown to be overexpressed in nearly all human carcinomas. ${ }^{6}$ In a recent study, it was shown that GLUT-1 is a major glucose transporter expressed in non-small-cell lung carcinoma, and the contribution of the other transporters such as GLUT-2, GLUT-3, GLUT-4 and GLUT-5 to the overall glucose metabolism in non-small-cell lung carcinomas appears to be minor. ${ }^{15}$ As only limited studies address the issue of GLUT-1 expression in lung carcinoma, we sought to investigate its expression and correlation with outcome in pulmonary neuroendocrine carcinomas.

\section{Materials and methods}

Pulmonary neuroendocrine carcinomas from the registry of the Department of Pulmonary and Mediastinal Pathology, Armed Forces Institute of Pathology, served as the basis for this study. Following institutional review board approval, the clinical and follow-up information and tissue material were collected and the tissue microarray was constructed as described earlier. ${ }^{16}$ The study cohort included 73 men and 83 women with a mean age of $59 \pm 15$ years (range, 19-83; median 62 years). Follow-up information was available for 122 cases with a median follow-up of 2 years. After identifying tumor area for each case from formalin-fixed paraffin-embedded tissue blocks, 0.6-mm cores were punched and embedded in the donor paraffin block. The tissue microarray block included 48 typical carcinoids, 31 atypical carcinoids, 27 large cell neuroendocrine carcinomas, 72 small cell carcinomas and 40 normal cores of lung parenchyma in duplicates. The core dropout rate was $10 \%$. An initial GLUT-1 antibody testing was carried out on a set of tumor cell lines and a set of surgical specimens of human placenta, pulmonary nonsmall-cell carcinomas and neuroendocrine carcinomas. The antibody showed diffuse staining with a breast carcinoma cell line, and a distinct membranous staining with placental cytotrophoblast and syncytiotrophoblast cells, erythrocytes, pulmonary adenocarcinoma, squamous cell carcinoma and small cell carcinoma but not with endothelial cells, respiratory epithelium, or connective tissue fibroblasts. After achieving satisfactory staining on the surgical specimens, the immunohistochemical studies were carried out on the tissue microarray section of pulmonary neuroendocrine carcinomas. Four-micron sections were deparaffinized in xylene and rehydrated through a series of graded alcohols. This was followed by antigen retrieval in $0.01 \mathrm{M}$ EDTA solution for $20 \mathrm{~min}$ at $100^{\circ} \mathrm{C}$. The slides were allowed to cool for $20 \mathrm{~min}$. After washing in PBS, endogenous peroxidase was blocked with $3 \%$ hydrogen peroxide for $10 \mathrm{~min}$, followed by incubation with PBS containing 10\% normal goat serum for $30 \mathrm{~min}$. The slides were then treated with polyclonal anti-GLUT-1 antibody (Dako, Carpinteria, CA, USA) at a 1:300 dilution overnight in a humidified chamber. Slides were rinsed with distilled water to remove the primary antibody. The secondary antibody was applied for $30 \mathrm{~min}$. Immunoreactions were detected by the labeled streptavidin-biotin-peroxidase complex technique and visualized using diaminobenzidine substrate for $10 \mathrm{~min}$, and counterstained with hematoxylin for cellular localization for $2 \mathrm{~min}$. Microscopic analysis of immunohistochemical study was performed under $\times 400$ magnification independently by three pulmonary pathologists. In the case of discrepant results, consensus was achieved with a second review. The intensity and the percentage of the stained tumor cells were evaluated. Only a distinct membranous staining was recorded. The intensity of GLUT-1 expression by the tumor cells was based upon comparison with the intensity of staining of the red blood cells in adjacent blood vessels. GLUT-1 red blood cell expression was assigned an intensity value of 2 . Tumor cells with no staining were considered negative, whereas the tumor cells that stained lighter than red blood cells were assigned a value of 1 . The tumor cells with equal or greater stain intensity were assigned values of 2 and 3, respectively. The mean overall score comprised the intensity score multiplied by the percent of positive tumor cells. This overall score was then averaged according to the number of available cores. If there was no tumor in a particular core, no score was given. Statistical analysis was performed using Statistical Software Package for the Social Sciences, version 13.0 for Windows (SPSS Inc., Chicago, IL, USA). Correlation of GLUT-1 expression with clinicopathological variables was performed by $\chi^{2}$ methods. Parametric tests were used to assess variables such as stage. One-way analysis of 
variance was used to test for differences among two or more independent groups and Kruskal-Wallis test was used for comparison of numerical data as appropriate. Kaplan-Meier plots and the log-rank test were used to investigate a potential relationship between GLUT-1 expression and patient survival. Univariate and multivariate analyses of potential prognostic factors were carried out by employing Cox proportional hazards modeling. A result was considered significant if the $P$-value was $<0.05$.

\section{Results}

Forty-seven percent (75/161) of pulmonary neuroendocrine carcinomas were immunoreactive with GLUT-1. A distinct membranous staining was found in $7 \%(3 / 46)$ of cases of typical carcinoid, $21 \%$ (6/29) of cases of atypical carcinoid, 74\% (17/23) of cases of large cell neuroendocrine carcinoma and $78 \%(49 / 63)$ of cases of small cell carcinoma. GLUT-1 expression was observed in neoplastic cells, preferentially along the luminal border of tumor islands or areas adjacent to necrosis (Figure 1). As expected, the background erythrocytes were positive for GLUT-1 in all the cases, whereas normal bronchial epithelium (4 cases), alveolar pneumocytes (40 cases), smooth muscle cells (2 cases), endothelial cells (40 cases) and connective tissue fibroblasts (10 cases) were negative. GLUT-1 expression positively correlated with tumor differentiation/tumor grade $(P<0.001)$, and with increasing age $(P=0.01)$ but not with gender, tumor size or stage (Table 1).
Univariate analysis of the prognostic significance of GLUT-1 showed that its expression was significantly associated with an elevated risk of death for neuroendocrine carcinomas as a group (risk ratio $=2.519 ; \quad 95 \% \quad$ confidence $\quad$ interval $=1.519$ 4.178; $P<0.001$ ) and carcinoids (risk ratio $=4.262$; $95 \%$ confidence interval $=1.472-12.343 ; P=0.01$ ). No prognostic correlations were observed when different levels of GLUT-1 expression or combined score were included in analysis. Survival curves generated by the Kaplan-Meier method also showed a statistically significant separation of GLUT-1positive vs GLUT-1-negative cases for all neuroendocrine carcinomas $(P<0.001)$ and carcinoids $(P=0.01)$ (Figure 2), but not for high-grade neuroendocrine carcinomas $(P=0.09)$. The curve separation was also seen for stage I-II $(P<0.001)$ but not for stage III-IV $(P=0.4)$ neuroendocrine carcinomas. In multivariate analysis, the patients' age $(<62$ years) (risk ratio $=2.693 ; 95 \%$ confidence interval $=1.207-6.009 ; P=0.02$ ), tumor differentiation/ grade (risk ratio $=2.144 ; 95 \%$ confidence interval $=1.367-3.362 ; P=0.001)$ and higher stage (risk ratio $=3.000 ; 95 \%$ confidence interval $=2.027$ $4.441 ; P<0.001)$ were independent adverse prognostic factors, but not GLUT expression (risk ratio $=0.536 ; \quad 95 \%$ confidence interval $=0.179$ 1.608; $P=0.27)$.

\section{Discussion}

GLUT-1 expression has been evaluated in varying carcinomas from different sites; $;^{7,8,11,17-20}$ however,

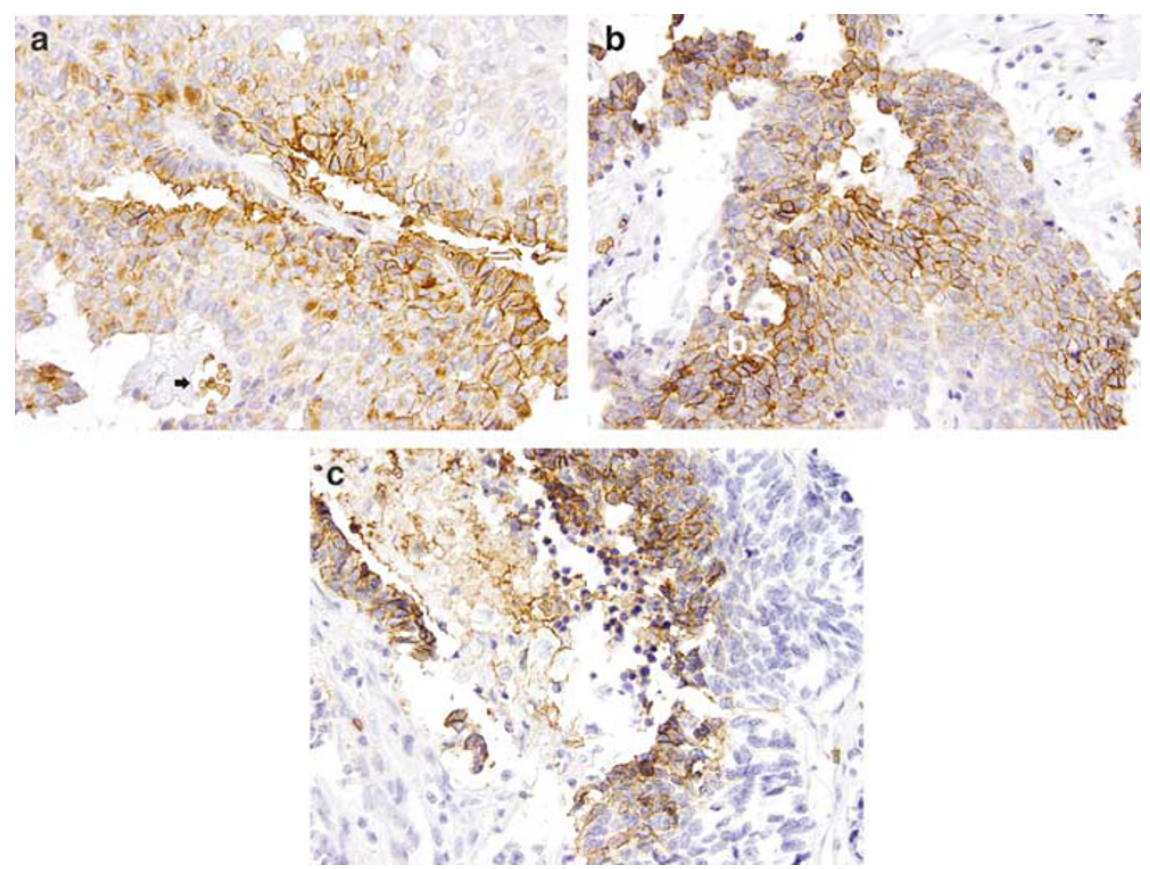

Figure 1 GLUT-1 expression in pulmonary neuroendocrine carcinomas. On immunohistochemical studies, GLUT-1 shows a distinctive membranous staining pattern (a, atypical carcinoid, $\times 400$ ); the bottom of the field (black arrow) shows a vessel with erythrocytes serving as an internal positive control. The staining is more prominent at the luminal borders of the tumor islands (b, large cell neuroendocrine carcinoma, $\times 400)$ or in the areas adjacent to necrosis $(\mathbf{c}$, small cell carcinoma, $\times 400)$. GLUT-1, glucose transporter-1. 
Table 1 Association of GLUT-1 expression with clinicopathologic variables

\begin{tabular}{|c|c|c|c|c|}
\hline Variables & $\begin{array}{l}\text { GLUT-1 } \\
\text { positive }\end{array}$ & $\begin{array}{l}\text { GLUT-1 } \\
\text { negative }\end{array}$ & $\mathrm{n}$ & P-value \\
\hline \multicolumn{5}{|l|}{ Age } \\
\hline$<62$ (median) & $35 \%$ & $65 \%$ & 71 & \multirow[t]{2}{*}{$0.01^{\mathrm{a}}$} \\
\hline$\geq 62$ (median) & $56 \%$ & $44 \%$ & 84 & \\
\hline \multicolumn{5}{|l|}{ Gender } \\
\hline Female & $51 \%$ & $49 \%$ & 83 & \multirow[t]{2}{*}{$0.57^{\mathrm{a}}$} \\
\hline Male & $43 \%$ & $57 \%$ & 73 & \\
\hline \multicolumn{5}{|l|}{ Tumor size } \\
\hline Mean \pm s.d. $(\mathrm{cm})$ & $3.2 \pm 1.8$ & $3.2 \pm 2.0$ & 130 & $0.92^{\mathrm{c}}$ \\
\hline \multicolumn{5}{|l|}{ Stage } \\
\hline I, II & $40 \%$ & $54 \%$ & 98 & \multirow[t]{2}{*}{$0.92^{\mathrm{b}}$} \\
\hline III, IV & $52 \%$ & $33 \%$ & 23 & \\
\hline \multicolumn{5}{|c|}{ Histology/differentiation } \\
\hline TC & $7 \%$ & $93 \%$ & 46 & \multirow[t]{4}{*}{$<0.001^{\mathrm{a}}$} \\
\hline $\mathrm{AC}$ & $21 \%$ & $79 \%$ & 29 & \\
\hline LCNEC & $74 \%$ & $26 \%$ & 23 & \\
\hline SCLC & $78 \%$ & $22 \%$ & 63 & \\
\hline
\end{tabular}

AC, atypical carcinoid; LCNEC, large cell neuroendocrine carcinoma; NEC, neuroendocrine carcinoma; SCLC, small cell lung carcinoma; TC, typical carcinoid.

${ }^{\mathrm{a}} \chi^{2}$ test.

${ }^{\mathrm{b}}$ Fisher's exact test.

${ }^{\mathrm{c}}$ Student's $t$-test.

little is known about its expression in neuroendocrine tumors. Ito et $a l^{21}$ studied the expression of GLUT isoforms in a range of pulmonary carcinomas and noted the presence of GLUT-1 in 7 of 11 small cell carcinomas. ${ }^{21}$ This was also confirmed by the detection of mRNA in a single case of small cell carcinoma and in a set of small cell carcinoma cell lines. ${ }^{21,22}$ Our study provides further evidence of frequent GLUT-1 expression in pulmonary small cell carcinoma $(78 \%)$ on a large number of cases. A novel finding in our study was also the detection of GLUT-1 in a significant percentage $(74 \%)$ of large cell neuroendocrine carcinoma. As a part of the neuroendocrine carcinoma spectrum, carcinoids fall into the low- or intermediate-grade end of the spectrum and in contrast to small cell carcinoma and large cell neuroendocrine carcinoma, they feature better prognosis, less frequent metastasis, lesser proliferative activity and longer mean doubling time. ${ }^{1}$ To the best of our knowledge, this study is the first documenting GLUT-1 expression in pulmonary carcinoids. As GLUT-1 upregulation is associated with an increased tumor metabolic rate, ${ }^{3}$ it is not surprising that we found a more frequent GLUT-1 expression in high-grade neuroendocrine carcinomas than in typical carcinoids and/or atypical carcinoids. It is reported that GLUT-1 expression correlates with grade in other lung tumors, in particular in adenocarcinomas, ${ }^{21,23}$ and carcinomas of breast ${ }^{24}$ and ovary. ${ }^{18}$ Similarly, we showed that GLUT-1 is seen throughout the entire spectrum of
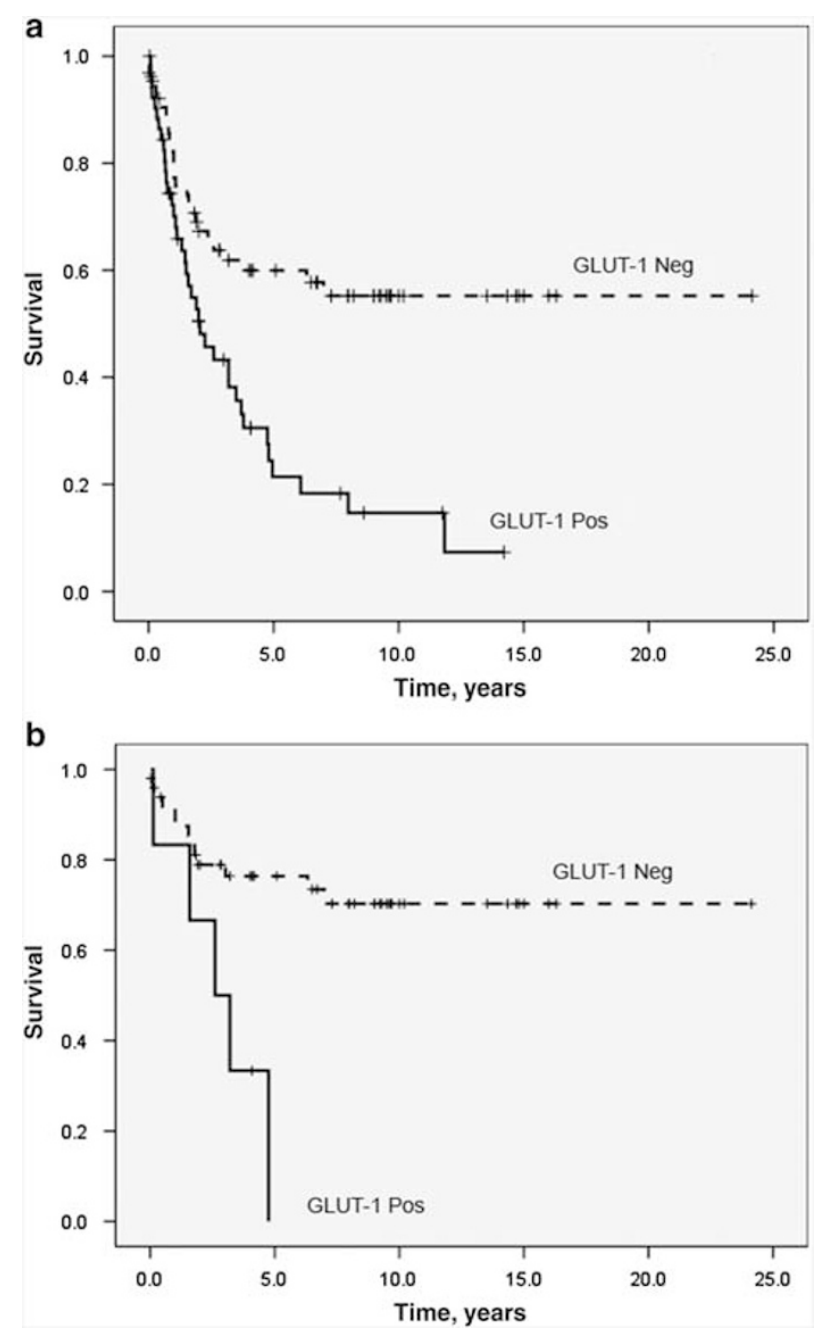

Figure 2 Overall survival analysis utilizing Kaplan-Meier method. There is a statistically significant curve separation between GLUT-1-positive and GLUT-1-negative cases for the whole group of neuroendocrine carcinomas $(\mathbf{a})(P<0.001)$ and carcinoids $(\mathbf{b})$ $(P=0.01)$. Pos, GLUT-1-positive cases; Neg, GLUT-1-negative cases. GLUT-1, glucose transporter-1.

neuroendocrine carcinomas, suggesting that this isoform plays an important role in glucose metabolism in these tumors. In turn, a consistent GLUT-1 expression in neuroendocrine carcinomas raises the question of whether it is also expressed in normal bronchiolar neuroendocrine cells. In the current set of cases, none of the bronchiolar cores from normal lung parenchyma controls (four cases, among which two cores had occasional neuroendocrine cells) showed GLUT-1 expression. Furthermore, none of the bronchioles/normal neuroendocrine cells from the tested resection specimens (five cases) showed expression of GLUT-1. These data and the absence of GLUT-1 expression in non-neoplastic tissue in lung and other organs $(6,19)$ suggest that normal neuroendocrine cells are negative for GLUT-1. Evaluation of GLUT-1 expression in hyperplastic neuroendocrine cells as seen in neuroendocrine hyperplasia associated with tumorlets/carcinoids or 
in the setting of diffuse idiopathic pulmonary neuroendocrine cell hyperplasia and/or in patients with chronic hypoxia as seen in cystic fibrosis or chronic interstitial lung disease appears as a plausible target for future studies.

A strong GLUT-1 immunostaining was shown in the hypoxic regions surrounding the necrotic foci of squamous cell carcinoma and adenocarcinoma of the lung. ${ }^{13,15,21,25}$ The direct effect of hypoxia on GLUT expression has been observed by increased GLUT-1 mRNA levels, the increased transcription of the GLUT-1 reporter gene, and the modulation of the GLUT-1 protein activity after induction of hypoxia. ${ }^{3}$ These prior immunohistochemical and molecular observations provide a good foundation to explain the pattern of GLUT-1 expression in neuroendocrine carcinomas. As observed in this study, the GLUT-1 expression was confined to the cellular membrane and was seen preferentially along the luminal borders of the tumor islands in neuroendocrine carcinomas. High-grade neuroendocrine carcinoma, especially, small cell carcinoma, is one of the most aggressive human malignancies, typically showing high tumor cell proliferation rates and infarct-like necrosis, and is bound to have frequent hypoperfused and/or hypoxic areas. In our study, small cell carcinoma had the most prominent luminal pattern of staining. This finding corresponds to that described in other tumors. Mamede et $a l^{25}$ found that immunoreactivity of GLUT-1 was mainly observed in the membrane of tumor cells and the expression of GLUT-1 localized preferentially at the edge of necrotic areas in non-small-cell carcinoma.

There is increased interest in identifying the association between GLUT-1 expression and prognosis in tumors. Haber et $a l^{19}$ showed that patients with colon carcinomas with greater than 50\% GLUT-1positive malignant cells had a higher mortality rate than patients whose tumors had less than $50 \%$ positivity. Similarly, GLUT-1 expression correlated with a less favorable outcome in studies of ovarian ${ }^{18}$ and breast ${ }^{20}$ carcinomas and non-small-cell carcinomas of the lung. ${ }^{13}$ The adverse prognostic significance of GLUT-1 expression was documented in our study as well. We found that within the whole spectrum of neuroendocrine carcinomas, the patients with GLUT1-positive tumors had a less favorable outcome than the patients with GLUT-1-negative tumors. Although this finding indicates a potential prognostic value of GLUT-1 for neuroendocrine carcinomas, it appears dependant on the tumor type and stage. When adjusted for tumor type/grade, GLUT-1 expression was also associated with unfavorable outcome for patients with carcinoids. However, as only approximately $12 \%$ of carcinoids showed GLUT-1 expression, the significance of its expression on prognosis in carcinoids may require further clarification employing a larger cohort.

In conclusion, similar to other tumors, GLUT-1 expression is seen in pulmonary neuroendocrine carcinomas showing a characteristic membranous pattern of staining and predilection for the luminal aspects of tumor islands. Its expression is observed throughout the entire spectrum of neuroendocrine carcinomas, correlates with the tumor differentiation/grade and likely indicates an important role for this protein in glucose metabolism in pulmonary neuroendocrine carcinomas.

\section{References}

1 Travis WD, Brambilla E, Muller-Hermelink HK, et al. Pathology \& Genetics: Tumours of the Lung, Pleura, Thymus and Heart. WHO International Histological Classification of Tumours. IARC Press: Lyon, France, 2004.

2 Simon GR, Wagner H. Small cell lung cancer. Chest 2003;123(1 Suppl):259S-271S

3 Macheda ML, Rogers S, Best JD. Molecular and cellular regulation of glucose transporter (GLUT) proteins in cancer. J Cell Physiol 2005;202:654-662.

4 Behrooz A, Ismail-Beigi F. Stimulation of glucose transport by hypoxia: signals and mechanisms. News Physiol Sci 1999;14:105-110.

5 Godoy A, Ulloa V, Rodriguez F, et al. Differential subcellular distribution of glucose transporters GLUT1-6 and GLUT9 in human cancer: ultrastructural localization of GLUT1 and GLUT5 in breast tumor tissues. J Cell Physiol 2006;207:614-627.

6 Younes M, Lechago LV, Somoano JR, et al. Wide expression of the human erythrocyte glucose transporter Glut1 in human cancers. Cancer Res 1996;56:1164-1167.

7 Chung JK, Lee YJ, Kim SK, et al. Comparison of [18F]fluorodeoxyglucose uptake with glucose transporter-1 expression and proliferation rate in human glioma and non-small-cell lung cancer. Nucl Med Commun 2004;25:11-17.

8 De SH, Landuyt W, Verbeken E, et al. The prognostic value of the hypoxia markers CA IX and GLUT 1 and the cytokines VEGF and IL 6 in head and neck squamous cell carcinoma treated by radiotherapy \pm chemotherapy. BMC Cancer 2005;5:42.

9 Khandani AH, Whitney KD, Keller SM, et al. Sensitivity of FDG PET, GLUT1 expression and proliferative index in bronchioloalveolar lung cancer. Nucl Med Commun 2007;28:173-177.

10 Laudanski P, Koda M, Kozlowski L, et al. Expression of glucose transporter GLUT-1 and estrogen receptors ERalpha and ER-beta in human breast cancer. Neoplasma 2004;51:164-168.

11 Palit V, Phillips RM, Puri R, et al. Expression of HIF1alpha and Glut-1 in human bladder cancer. Oncol Rep 2005;14:909-913.

12 Wincewicz A, Sulkowska M, Koda M, et al. Clinicopathological significance and linkage of the distribution of HIF-1alpha and GLUT-1 in human primary colorectal cancer. Pathol Oncol Res 2007;13:15-20.

13 Younes M, Brown RW, Stephenson M, et al. Overexpression of Glut1 and Glut3 in stage I nonsmall cell lung carcinoma is associated with poor survival. Cancer 1997;80:1046-1051.

14 Clavo AC, Brown RS, Wahl RL. Fluorodeoxyglucose uptake in human cancer cell lines is increased by hypoxia. J Nucl Med 1995;36:1625-1632. 
15 Brown RS, Leung JY, Kison PV, et al. Glucose transporters and FDG uptake in untreated primary human non-small cell lung cancer. J Nucl Med 1999;40:556-565.

16 Fukuoka J, Dracheva T, Shih JH, et al. Desmoglein 3 as a prognostic factor in lung cancer. Hum Pathol 2007;38:276-283.

17 Airley R, Loncaster J, Davidson S, et al. Glucose transporter glut-1 expression correlates with tumor hypoxia and predicts metastasis-free survival in advanced carcinoma of the cervix. Clin Cancer Res 2001;7:928-934.

18 Cantuaria G, Fagotti A, Ferrandina G, et al. GLUT-1 expression in ovarian carcinoma: association with survival and response to chemotherapy. Cancer 2001;92:1144-1150.

19 Haber RS, Rathan A, Weiser KR, et al. GLUT1 glucose transporter expression in colorectal carcinoma: a marker for poor prognosis. Cancer 1998;83: 34-40.

20 Kang SS, Chun YK, Hur MH, et al. Clinical significance of glucose transporter 1 (GLUT1) expression in human breast carcinoma. Jpn J Cancer Res 2002;93:1123-1128.

21 Ito $\mathrm{T}$, Noguchi $\mathrm{Y}$, Satoh S, et al. Expression of facilitative glucose transporter isoforms in lung carcinomas: its relation to histologic type, differentiation grade, and tumor stage. Mod Pathol 1998;11:437-443.

22 Pedersen MW, Holm S, Lund EL, et al. Coregulation of glucose uptake and vascular endothelial growth factor (VEGF) in two small-cell lung cancer sublines in vivo and in vitro. Neoplasia 2001;3:80-87.

23 Higashi K, Ueda Y, Sakurai A, et al. Correlation of Glut-1 glucose transporter expression with $\left[{ }^{18} \mathrm{~F}\right] \mathrm{FDG}$ uptake in non-small cell lung cancer. Eur J Nucl Med 2000;27:1778-1785.

24 Younes M, Brown RW, Mody DR, et al. GLUT1 expression in human breast carcinoma: correlation with known prognostic markers. Anticancer Res 1995;15:2895-2898.

25 Mamede M, Higashi T, Kitaichi M, et al. [18F]FDG uptake and PCNA, Glut-1, and Hexokinase-II expressions in cancers and inflammatory lesions of the lung. Neoplasia 2005;7:369-379. 